\title{
PARP1 and PARP2 affinity to the lessions in the context of nucleosomes
}

\author{
Tatiana Andreevna Kurgina \\ LBCE \\ Institute of Chemical Biology and \\ Fundamental Medicine SB RAS \\ Novosibirsk, Russia \\ t.a.kurgina@gmail.com \\ Rashid Oktamovich Anarbaev \\ $\mathrm{LBCE}$ \\ Institute of Chemical Biology and \\ Fundamental Medicine SB RAS \\ Novosibirsk, Russia \\ ranarbaev@gmail.com
}

\author{
Ekaterina Anatolyevna Belousova \\ LBCE \\ Institute of Chemical Biology and \\ Fundamental Medicin SB RAS \\ Novosibirsk, Russia \\ rina@niboch.nsc.ru \\ Olga Ivanovana Lavrik \\ LBCE \\ Institute of Chemical Biology and \\ Fundamental Medicine SB RAS \\ Novosibirsk, Russia \\ lavrik@niboch.nsc.ru
}

\author{
Michail Michailovich Kutusov \\ LBCE \\ Institute of Chemical Biology and \\ Fundamental Medicin SB RAS \\ kutuzov.mm@mail.ru
}
Svetlana Nikolaevna Khodireva LBCE
Institute of Chemical Biology and Fundamental Medicine SB RAS Novosibirsk, Russia svetakh@niboch.nsc.ru

\begin{abstract}
Members of Poly(ADP-ribose)polymerase family are key enzymes regulating the DNA repair system. For example, PARP1 binds damaged DNA resulting catalytically active complex. Using NAD+ as a substrate PARP1 catalyzes the synthesis of poly(ADP-ribose) covalently attached to many target proteins, including itself. This enzyme is a promising target for creating anti-cancer drugs. However, these compounds often display high cytotoxicity. These problems require new inhibitors as well as testing approaches. The inhibitors verification is additionally obstructed by complex chromatin organization of DNA in cells. The use of short DNA makes it difficult to extrapolate the results obtained in vitro to in vivo conditions. In the current work we present a test system that allows real time analyzing of PARP1 DNA binding in nucleosome context.
\end{abstract}

Keywords - poly(ADP-ribosyl)ation, nucleosome, DNA repair.

\section{Introduction}

Genome stability in higher eukaryotes is supported by the activity of several DNA repair pathways, which depend on the type of damage. Poly(ADP-ribose)polymerases 1 and 2 (PARP1 and PARP2) are some of the key enzymes regulating DNA repair system. This enzymes functions as a "sensor" of DNA damage. The interaction with damaged DNA stimulates the NAD+-dependent poly(ADPribosyl)ation PARPs activity. The polymer of poly(ADPribose) has a negative charge and its covalent attachment to PARPs leads to the dissociation of poly(ADP-ribosyl)ated PARPs from DNA due to electrostatic repulsion. The poly(ADP-ribose) is a signal molecule for the enzymes and cellular systems concerning DNA damage.

Recently, we created a method that can be used in real time measurements [1]. PARP1 was activated with DNA duplexes containing damage in one strand and fluorophore at the 3'-end of the other strand. Fluorescent label is necessary for detection of the fluorescence anisotropy. Fluorescence anisotropy was defined as the ratio of the polarized component to the total intensity: $A=(\mathrm{I} 1-\mathrm{I} 2) /(\mathrm{I} 1+$ 2I2), where I1 and I2 are the intensities of the light emitted by a fluorophore along different axes of polarization. The anisotropy level was used to estimate the size of the complex containing fluorescent DNA.
In this work, we applied this method to the nucleosome core particle (NCP). This system is more relative to in vivo conditions than naked DNA. In this system, anisotropy increases when PARP proteins bind to the nucleosome in the immediate vicinity of the FAM-labeled DNA ends.

\section{Results}

In this study, we used 3 different nucleosome structures and 3 different DNA structures. First, DNA and NCP without anything lesions (except blunt DNA-ends) were used. Further, we use two lesion-intermediates of base excision repair: AP-site and gap. We evaluated dissociation constants $(\mathrm{Kd})$ for PARP1 and PARP2 on this structures.

We did not detect any difference between affinity to native and damage nucleosomes. It can be explained by strong affinity of PARP1 to blunt DNA ends: at the all cases, PARP1 bind DNA ends and direct influence on the fluorophore mobility.

The dramatic decreasing of $\mathrm{Kd}$ for the PARP2 and gaped-nucleosome is shown. This data are consistent with previous studies [2] of PARP2 interaction with DNA: Kd for single strand break is lower than Kd for blunt DNA end.

\section{Conclusions}

We have shown the applicability of our spectrophotometry method to detect PARP1 and PARP2 interaction with NCP. We detected binding of these enzymes to different lesions with different affinity in the context of nucleosome.

\section{ACKNOWLEDGMENT}

The work was supported by the grant from RFFS 17-0000097.

\section{REFERENCES}

[1] Kurgina T.A., Anarbaev R.O., Sukhanova M.V. and Lavrik O.I. "A rapid fluorescent method for the real-time measurement of poly(ADP-ribose) polymerase 1 activity", Anal Biochem., vol. 15, pp. 91-97, March 2018.

[2] Sukhanova M.V., Abrakhi S., Joshi V., Pastre D., Kutuzov M.M., Anarbaev R.O., Curmi P.A., Hamon L. and Lavrik O.I. "Single molecule detection of PARP1 and PARP2 interaction with DNA strand breaks and their poly(ADP-ribosyl)ation using highresolution AFM imaging.", Nucleic Acids Res, vol. 7, April 2016. 